\title{
High-Temperature Constitutive Equations of Q235 Galvanized Sheet
}

\author{
Sunyang Zhou ${ }^{1, a}$, Huihui Zou ${ }^{1, b}$ and Xintang Wang ${ }^{1, c}$ \\ 1'Department of Civil Engineering, Ningbo University, Zhejiang, Ningbo 315211, China \\ awxt196322@126.com, bhuihui@nbu.edu.cn, ‘wangxintang@nbu.edu.cn
}

\begin{abstract}
Keywords: high temperature, constitutive equations, fitting, Q235 thin-walled galvanized sheet Abstract. Based on a series of steady tensile tests and transient tensile tests of Q235 thin-walled galvanized sheet with depth of $2 \mathrm{~mm}$ in elevated temperature using high temperature furnace, the formulas of high temperature mechanical property indexes and high temperature constitutive equations of the material were obtained. It was seen that the material had an obvious period of yielding when it was axially stretched at low temperature, however it didn't yield any more above $300^{\circ} \mathrm{C}$. The formula of the high temperature constitutive equations presented here can be used for fire-resistance computation of the structure constructed with the material.
\end{abstract}

\section{Introduction}

As for the steel structure building, anticorrosion and fireproofing are two of the most vital aspects of ensuring the safety of the structure. Kankanamge [1] has studied high-temperature mechanical properties of the cold-formed thin-walled steel structures with two kinds of thickness and strength grade. Ranawaka [2] has studied high-temperature mechanical properties of the G250 and G550 thin-walled galvanized sheet with thickness of $0.6 \mathrm{~mm}, 0.8 \mathrm{~mm}$ and $0.95 \mathrm{~mm}$. Huang [3] has made experiment of high-temperature mechanical properties of the thin plate materials of cold-formed molding square steel tube, whose results are compared with the present design method. Yan [4] has made the experiments of mechanical property and bearing capacity of the connection of cold-formed thin-walled steel structures.

But the hot dip galvanized sheet made in China is not involved in all these researches, and the relationship of stresses and strains presented in these researches has a certain application conditions. Thus, based on a series of steady high-temperature property tests and transient high-temperature property tests of Q235 thin-walled galvanized sheet made in China, the high-temperature mechanical property indexes of the galvanized sheet with thickness of $2 \mathrm{~mm}$ were presented, which may be applied to the fire-resistance design of cold-formed thin-walled steel structures and improvement of relevant design specification.

\section{Experimental Scheme}

Materials and Equipment of the Experiment The material involved in high-temperature tensile experiment is Q235 hot-dipped galvanized sheet with thickness of $2 \mathrm{~mm}$. The loading equipment used in the experiment is WAW-600C microcomputer-controlled electro-hydraulic servo universal testing machine. Its maximum testing force is $600 \mathrm{kN}$ and its precision of testing force is $\pm 1 \%$. The measurement and control software adopted in this testing machine is STS2011 software. Data is analyzed by the means of human-computer interaction and processed by the requirement of

《Tensile test method of metal material in room-temperature》(GB/T 228-2002). The heating and temperature-control equipment used in this experiment is GW-1200B high-temperature furnace produced in Changchun Fangrui Science Company Limited in China. The heating and temperature-control equipment consists of high-temperature furnace, GW-1200B controlling box, high-temperature rod, high-temperature collet, dial gauge and rotating arm.

Testing Scheme According to national standard 《Tensile test method of metal in high-temperature》 (HB5195-1996) and 《Tensile test method of room-temperature metal》 (HB5143-1996), the loading condition of the specimens is: heating velocity is kept as $10 \sim 15^{\circ} \mathrm{C} / \mathrm{min}$; the target temperature muat be kept for 15 minutes before the tensile test for the constant temperature loading test; 
strain velocity should be about $0.005 / \mathrm{min}$ when the specimen is drawn. In order to obtain the exact test data, two sets of specimens are made, and steady tests together with transient tests are finished separately. The temperature level is divided into 12 temperature points, that is $20^{\circ} \mathrm{C}$. $150^{\circ} \mathrm{C} 、 200^{\circ} \mathrm{C} 、 250^{\circ} \mathrm{C} 、 300^{\circ} \mathrm{C} 、 350^{\circ} \mathrm{C} 、 400^{\circ} \mathrm{C} 、 450^{\circ} \mathrm{C} 、 500^{\circ} \mathrm{C} 、 550^{\circ} \mathrm{C} 、 600^{\circ} \mathrm{C}$ and $700^{\circ} \mathrm{C}$, totally.

\section{Experimental Results and Analysis}

Test Phenomenon For Q235 galvanized sheet, a set of specimens at every temperature point from $20^{\circ} \mathrm{C}$ to $800^{\circ} \mathrm{C}$ were respectively tested, and the main features are described as:

(1) At $500^{\circ} \mathrm{C}$ to $600^{\circ} \mathrm{C}$, the surface feature of specimen after cooling doesn't have the original luster and the surface appears a little dark and the zinc coat nearly disappears. The fracture is completely black and there is no metallic luster. Necking phenomenon of the place where the specimen is damaged is quite evident and the shrinking percentage of fracture is larger.

(2) At $700^{\circ} \mathrm{C}$, the surface of specimen has no mental luster at all and appears a little yellow because of high-temperature firing, and the color of the specimen's surface closer to the damaged section is getting much yellower. The fracture is black, the shrinking percentage of which is extremely notable. Necking phenomenon is more evident and the elongation percentage of specimen is larger when it is damaged.

Results of High-temperature Tensile Property Test and Analysis The test results of elastic modulus, yielding strength and ultimate strength were drawn in Figure 1.

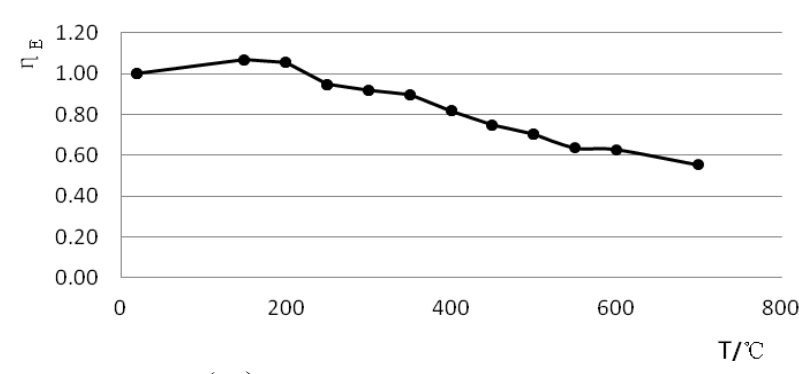

(a) Elastic modulus

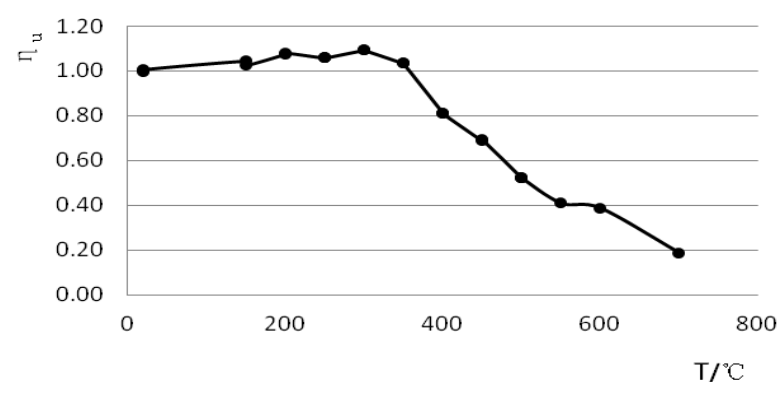

(c) Ultimate strength

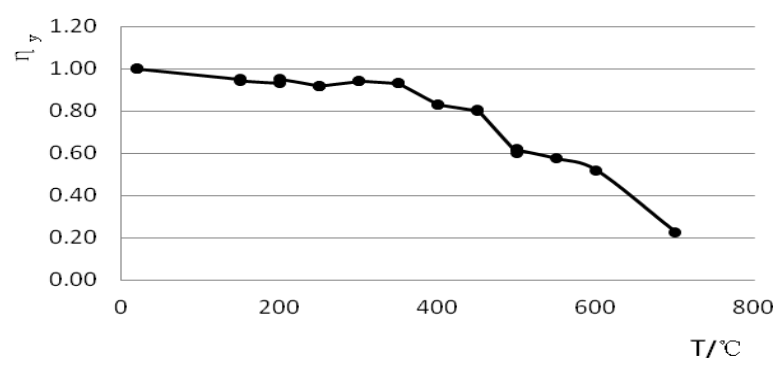

(b) Yielding strength

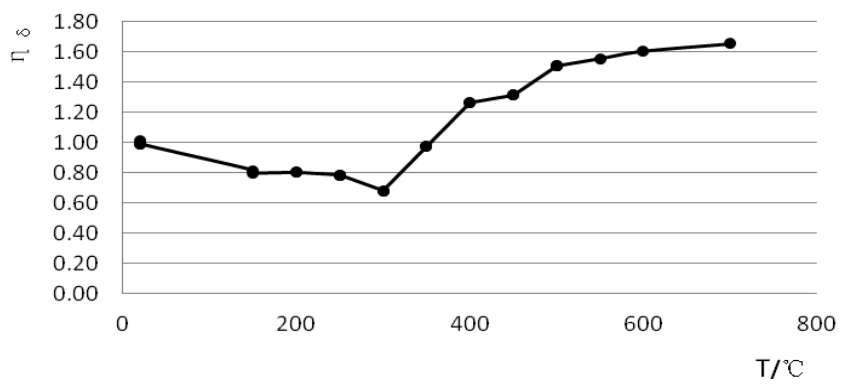

(d) Elongation percentage

Figure 1 Variation of reduction coefficients of mechanical properties with temperature

It was seen from the results shown in Figure 1 that these three mechanical indexes showed a declined tendency At $300^{\circ} \mathrm{C}$ to $700^{\circ} \mathrm{C}$, which got much rapider above $450^{\circ} \mathrm{C}$, which meant that the bearing capacity of the specimen had gradually lost and its stress-strain curve did not have an obvious yielding phase any more.

\section{Fitting of Test Results and Constitutive Model}

Fitting of Test Results According to the test results presented here, the formula of high-temperature mechanical property indexes of Q235 galvanized sheet can be fitted as: 
(1) Elastic modulus

$$
E_{T} / E=-2.504 \times 10^{-15} T^{5}+8.02 \times 10^{-9} T^{3}-7.659 \times 10^{-6} T^{2}+0.00142 T+0.9807
$$

Where $E_{T}$ is the elastic modulus of galvanized sheet at temperature of T. $E$ is the elastic modulus of galvanized sheet at normal temperature. $T$ is the temperature, the unit of which is ${ }^{\circ} \mathrm{C}$.

(2) Yield strength

$$
f_{y T} / f_{y}=-1.808 \times 10^{-9} T^{3}-3.181 \times 10^{-7} T^{2}-4.899 \times 10^{-6} T+0.989
$$

Where $f_{y T}$ is the yield strength of this material at temperature of T. $f_{y}$ is the yield strength of this material at normal temperature.

(3) Elongation percentage

$$
\delta_{u T} / \delta_{u}=-1.638 \times 10^{-8} T^{3}+2.026 \times 10^{-5} T^{2}-0.00538 T+1.124
$$

In which $\delta_{u T}$ is the elongation percentage of this material at temperature of $\mathrm{T}$, and $\delta_{u}$ is the elongation percentage of this material at normal temperature.

The fitting formulas of mechanical property indexes presented above were plotted together with the test results as shown in Figure 2. It is seen that the fitting curves fit the testing results very well.

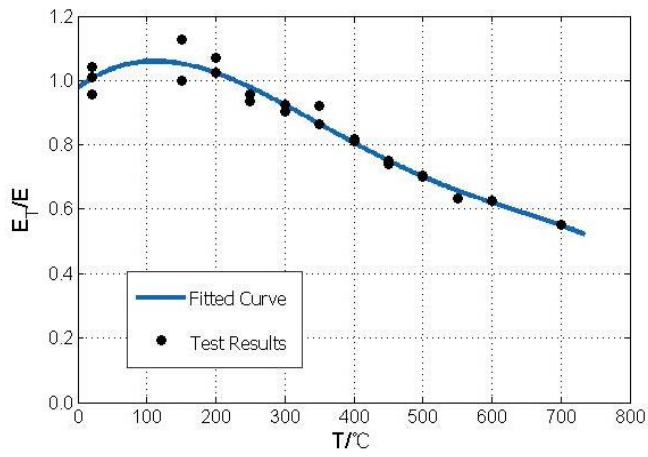

(a)Elastic modulus

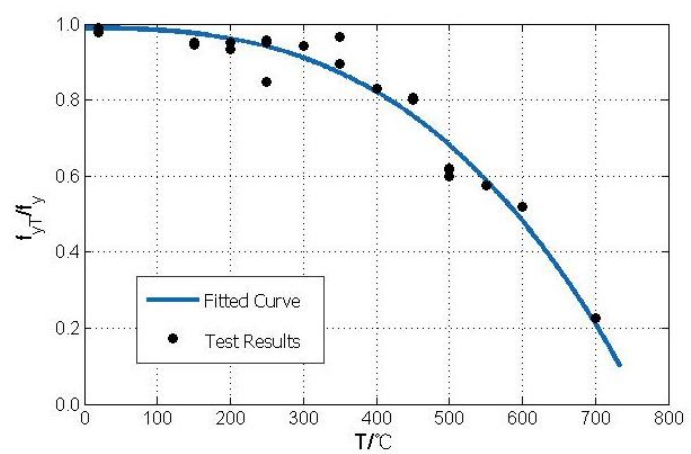

(b) Yield strength

Figure 2 Fitted curves and experimental results

Constitutive Model Referring to the steel three-polyline model put forward in existing papers, the constitutive relation of Q235 galvanized sheet at high temperature was obtained in this paper as:

$$
\begin{aligned}
& \sigma_{T}=\left\{\begin{array}{cc}
E_{T} \varepsilon_{T} & \left(\varepsilon_{T} \leq f_{p T} / E_{T}\right) \\
f_{p T}+E_{t T}\left(\varepsilon_{T}-f_{p T} / E_{T}\right) & \left(f_{p T} / E_{T}<\varepsilon_{T} \leq \varepsilon_{y T}\right. \\
f_{y T} & \left(\varepsilon_{T}>\varepsilon_{y T}\right)
\end{array}\right. \\
& \varepsilon_{y T}=f_{y T} / E_{t T}+f_{p T} / E_{T} \\
& f_{p T}=\left\{\begin{array}{lc}
f_{y T} & T<200^{\circ} \mathrm{C} \\
(1.4-0.002 T) f_{y T} & 200^{\circ} \mathrm{C} \leq \mathrm{T} \leq 450^{\circ} \mathrm{C} \\
0.5 f_{y T} & T>450^{\circ} \mathrm{C}
\end{array}\right. \\
& E_{t T}=\beta E_{T}
\end{aligned}
$$




$$
\beta=\left\{\begin{array}{cl}
0 & \multicolumn{1}{c}{T \leq 200^{\circ} \mathrm{C}} \\
-1.675 \times 10^{-10} T^{4}+2.787 \times 10^{-7} T^{3} & \\
-1.681 \times 10^{-4} T^{2}+4.314 \times 10^{-2} T-3.867 & 200^{\circ} \mathrm{C}<T \leq 600^{\circ} \mathrm{C} \\
0 & T>600^{\circ} \mathrm{C}
\end{array}\right.
$$

In which $E_{t T}$ is the elastic modulus of material in the period of softening at temperature of T. $\beta$ is the material softening coefficient of elastic modulus. The physical meanings of these parameters are shown in Figure 3. $E_{T}$ and $f_{y T}$ are calculated according to Eq. 1 and Eq. 2.

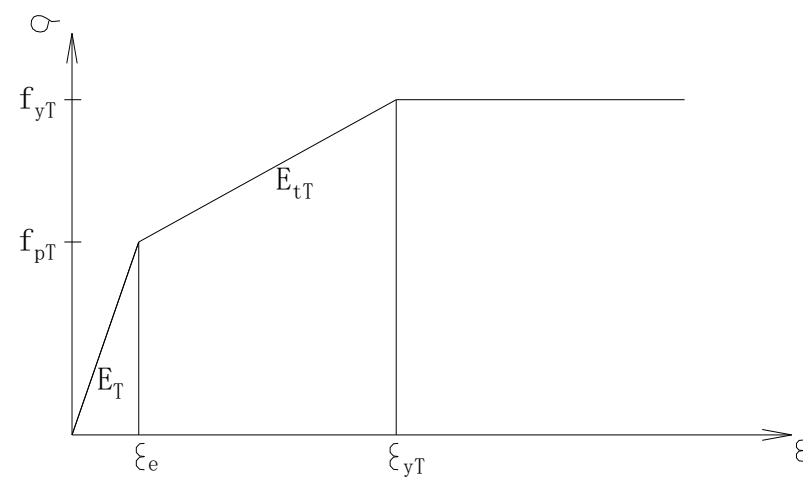

Figure 3 Three-line model of Q235 steel sheet

\section{Conclusions}

The main conclusions were reached as : (1) The material has an obvious period of yielding when it is axially stretched at low temperature, however it doesn't yield any more above $300^{\circ} \mathrm{C}$. (2)The formulas of mechanical property indexes established in this paper fit the experimental results very well. (3)The formulas of mechanical property indexes and relationship of stress and strain can be applied to the fire-resistance analysis of Q235 thin-walled galvanized sheet and reference of drawing up of relevant design specification.

\section{Acknowledgements}

This work was financially supported by the Applied Research Project of Public Welfare Technology of Zhejiang Province (2017C33013)

\section{References}

[1] N. D. Kankanamge, M. Mahendran: Thin-Walled Structures, Vol. 49(2011), p.26.

[2] T. Ranawaka, M. Mahendran: Fire Safety Journal Vol. 44 (2009), p. 219.

[3] Y. Huang, B. Young: Journal of Constructional Steel Research, Vol.92 (2014), 92, p.103.

[4] Y. Shu, B. Young: Engineering Structures, Vol.35 (2012), p.228.

[5] J.C. Zhao, Z.Y. Shen: Industry Construction, Vol.9(1996), p.2. (in Chineses) 